\title{
PARA UN ANÁLISIS CRÍTICO DEL CONCEPTO DE NORMALIZACIÓN FILOSÓFICA
}

\author{
Juan Camilo Betancur* \\ doi:10.11144/Javeriana.uph32-65.acnf
}

\section{RESUMEN}

El concepto de "normalización filosófica" se ha convertido en un lugar común poco cuestionado en la narración del proceso histórico de la filosofía en Latinoamérica. A partir de la teoría de los estratos del tiempo planteada por R. Koselleck, el presente trabajo propone una revisión crítica de dicho concepto.

Palabras clave: filosofía latinoamericana; normalidad filosófica; historia de la filosofía; historia de los conceptos

* Colegio Campoalegre, Sopó, Colombia.

Correo electrónico: juancamilo_betancur@yahoo.com

Para citar este artículo: Betancur, J.C. (2015). Para un análisis crítico del concepto de normalización filosófica. Universitas Philosophica, 32(65), pp. 137-158. ISSN 0120-5323, ISSN en línea: 2346-2426, doi:10.11144/ Javeriana.uph32-65.acnf

El presente artículo surge a partir de la ponencia presentada en el V Encuentro de investigadores organizado por la Universidad Santo Tomás el 23 de agosto 2012. 


\title{
FOR A CRITICAL ANALYSIS OF THE CONCEPT OF PHILOSOPHICAL NORMALIZATION
}

\author{
Juan Camilo Betancur
}

\begin{abstract}
The concept of "philosophical normalization" has become common place, recently questioned in the narrative of the historical process of philosophy in Latin America. From the theory of layers of time raised by R. Koselleck this paper proposes a critical review of this concept.

Key Words: Latin American philosophy; philosophical normalization; history of philosophy; history of concepts
\end{abstract}


EL CONCEPTO DE "NORMALIDAD" (o "normalización") ha sido profusamente empleado en las últimas décadas para describir y periodizar el proceso de la filosofía en Colombia. Se ha señalado con él la ruptura con los modos previos de hacer filosofía y el momento de introducción de la filosofía moderna a este país. Pero este uso del concepto se ha venido convirtiendo en un lugar común, poco o nada cuestionado, y cuyo énfasis recae en el aspecto institucional de la normalización: la consolidación de la filosofía como oficio disciplinar que comenzó a gozar de autonomía temática e investigativa desde el segundo tercio del siglo XX (Sierra, 1985; Botero, 2009; Pachón, 2011). Con ello, como veremos, se ha soslayado lo que pudo haber sido el verdadero aporte de esta época a varios temas y problemas que todavía conciernen a la reflexión filosófica en Colombia.

Recordemos que el término "normalización" proviene del concepto de "normalidad filosófica”, usado en el contexto más general de Latinoamérica, desde que Francisco Romero lo acuñó en el segundo tercio del siglo XX. En años recientes, algunas críticas al concepto han mostrado los riesgos que entraña su uso irreflexivo. Raúl Fornet-Betancourt, por ejemplo, señala que la categoría de "normalidad filosófica" supone una determinada concepción de filosofía que no estamos obligados a compartir y que, más bien, implica varios sesgos para el estudio de la producción filosófica en Latinoamérica (Fornet, 2000, pp. 121-122, 125). El concepto termina centrando la importancia de los "fundadores de la normalidad" en su aporte a la filosofía académica producida con la institucionalización universitaria (Fornet, 2000, p. 124). Siendo el filósofo profesional un problema abierto desde Sócrates, el riesgo radica en que esta figura se vuelva excluyente de otras formas de ejercicio filosófico (Fornet, 2000, p. 129). Así pues, la categoría de normalidad termina descalificando la filosofía anterior al hablar de una generación pionera que por primera vez filosofa, excluye respecto de su propio presente aquellos pensadores que estén al margen de la "normalidad" por haber practicado filosofía desde otros cánones de producción (Fornet, 2000, p. 127), y potencia un proyecto monocultural de filosofía; hacer filosofía "a la europea" (Fornet, 2000, pp. 128-129).

Riesgos semejantes se presentan para la investigación histórica de la filosofía en Colombia. El uso no cuestionado del concepto de normalización le ha dado más énfasis al aspecto institucional de la filosofía. Así, no solo se tiende a desconocer la 
obra de pensadores previos a la "normalización", como Carlos Arturo Torres ${ }^{1}$, Luis López de Mesa o Fernando González, sino también de pensadores contemporáneos y en algún sentido "marginales" a ella, como Julio Enrique Blanco, Joaquín Vallejo Arbeláez, Jaime Jaramillo Uribe, Estanislao Zuleta, Nicolás Gómez Dávila, entre otros. Algunos estudios recientes han señalado la importancia de profundizar en la investigación sobre estos autores, pero todavía les asignan un lugar marginal (Pachón, 2011, p. 125), pues los valoran en función de su aporte al proceso de normalización. Esto acarrea una visión demasiado estrecha de lo que pueda considerarse como historia de la filosofía en Colombia, pues se descarta la obra de un amplio grupo de pensadores que no fueron profesores o escritores de filosofía, pero que plantearon problemas sobre la sociedad, la religión, la política, el derecho, la historia, la antropología, la ciencia, en permanente interlocución con la filosofía.

Incluso dentro del estrecho campo del proceso de institucionalización, veremos que los filósofos universitarios han sido presentados como simples repetidores devotos sin producción original, aislados de su propia realidad social, como si respondieran a meras cuestiones exegéticas del todo irrelevantes. Los autores reconocidos por su "aporte" al proceso normalizador, como Rafael Carrillo, Cayetano Betancur o Danilo Cruz Vélez, han sido presentados como la "generación normalizadora", "los inauguradores" o "pioneros de la filosofía moderna en Colombia”, pero su estudio y exposición se ha hecho como en beneficio de inventario, resaltando apenas su aporte institucional reflejado en la fundación de facultades o revistas filosóficas y, en suma, en la consolidación del oficio profesoral de la filosofía. Quizá estos problemas en el concepto de normalización no solo hayan

1 Sobre Carlos Arturo Torres es interesante ver, por ejemplo, los dos lugares históricos que le ha dado el profesor Sierra Mejía. Mientras en 1982 lo ponía al margen del proceso de normalización, por no haber en él un pensamiento rigurosamente filosófico o centrado en la filosofía, en 2010 dejará de lado el concepto de normalización para hablar más bien de "solidificación del trabajo filosófico" (Sierra, 2010, p. 290) y pondrá a Torres como auténtico representante de una tradición de un modo de trabajo filosófico, no exegético, sino atento del uso instrumental de la filosofía, en función de problemas locales (Sierra, 2010, p. 289). Interesa notar también cómo el profesor Marquínez Argote incluye a Torres, González y López de Mesa en la categoría de la "generación de los fundadores", por compartir con ella los ideales de un pensamiento propio y original (Marquínez, 2005: p. 18). Sobre la base de esta inclusión podría hallarse un concepto analógico de "filosofía latinoamericana" en virtud del cual, más allá de la normalización en un sentido institucional, Marquínez le da un lugar fundacional a estos autores. 
determinado la narración de la historia de la filosofía sino, además, la experiencia misma de hacer filosofía en Colombia hoy.

El presente ensayo propone llenar el anterior vacío a través de una crítica del concepto de "normalización filosófica". La crítica de Fornet-Betancourt revela cómo las etapas previas, las contemporáneas y las posteriores al período de que hablaba Romero, han sido interpretadas en función de este concepto de normalidad. Su método de trabajo para emprender esta crítica consiste en contrastar la historiografía escrita, esto es, la versión romeriana, con las fuentes primarias, que son la obra misma de los llamados "normalizadores" (Fornet, 2000, p. 126). Así, Fornet-Betancourt abre el campo para una reescritura de esta historia. Otro tanto se puede hacer con los pensadores colombianos que han sido ya archivados y catalogados, con sospechosa reverencia, como "la generación normalizadora". Haré uso de algunas claves metodológicas propuestas por la Teoría de los estratos del tiempo de Reinhart Koselleck, para sentar las bases de una reescritura y continuación de la historia que puedan dar cuenta del proceso de esta filosofía en el siglo XX. Mi tesis de trabajo apunta a proponer la posibilidad de realizar un análisis crítico de la historia del concepto de normalización, a partir de tres estratos temporales propuestos por Koselleck. Así, en un primer momento, resumiré algunas tesis de los estratos del tiempo para que, en un segundo momento, su aplicación cobre sentido en la historiografía de la filosofía en Colombia.

1. Una teoría de la historia para la historia de la filosofía en Colombia

La pregunta básica por las estructuras del tiempo debe hacer posible formular preguntas especificamente históricas. Koselleck, Futuro pasado, p. 128

SI QUEREMOS ENTENDER LAS CONDICIONES que han hecho posible la escritura de una historia, tendremos que prestar atención a los cambios en dicha experiencia histórica. Koselleck busca plantear tal correspondencia entre cambios metodológicos y cambios de experiencia, en cuanto ambos son posibles por ciertas condiciones antropológicas comunes (Koselleck, 2001, p. 47) que son el contenido de unas estructuras duraderas de largo plazo, es decir, de modos de la experiencia humana que preceden formalmente a toda adquisición concreta de experiencia 
y cuya repetibilidad hace posible concebir la historia (Koselleck, 2001, p. 81) $)^{2}$. Koselleck propondrá su Teoría de los estratos del tiempo para dar cuenta de esos modos estructurales de la experiencia que nos servirán como criterio de análisis de cualquier historia: es decir, para separar distintas historias y métodos históricos según su alcance temporal ${ }^{3}$.

Ahora bien, el concepto de historia es ambiguo: designa tanto la experiencia de la realidad como la investigación metódica de esa experiencia (Koselleck, 2001, pp. 47-49). Debido a esa doble significación del concepto de historia, la teoría de Koselleck se expone en dos momentos: primero, analiza las vivencias que determinan los cambios en la experiencia; después, relaciona este análisis con los cambios de método de la ciencia histórica. Del primer momento reseñaré sus tesis sobre la constitución temporal de la experiencia. El segundo lo expondré en la segunda parte de este trabajo, como aplicación al análisis de la "normalización filosófica".

2 Cabe aclarar que la propuesta teórica de Koselleck se mueve en un marco de inspiración kantiana, en cuanto va tras los modos formales de la experiencia que determinan el ámbito de la experiencia histórica posible. Como veremos, más que tratarse de una semejanza superficial de terminologías, sorprende que Koselleck determine el ámbito de la experiencia histórica, no desde la discusión sobre la relación entre sujeto y objeto, fenómeno y noúmeno -como lo intentó Dilthey- (Palti, 2001, pp. 11-14), sino desde un análisis temporal de la experiencia, de modo semejante a como lo hizo Kant en las "Analogías de la experiencia" (a partir de aspectos formales de la intuición interna). En esta misma línea se podría comprender también cómo los conceptos históricos, en cuanto constitutivos de la experiencia, se cargan también de modos temporales desde los cuales han de ser analizados. Se mantiene, pues, un fuerte parentesco con los desarrollos kantianos del tiempo, ya con su análisis de la posible aplicación de las categorías a las intuiciones (Esquematismo trascendental), ya en el uso de estas categorías para constituir los ámbitos de la naturaleza y la experiencia (Analogías de la experiencia). La presente exposición se concentrará, sin embargo, solo en la propuesta formal de los modos de la experiencia, teniendo que dejar para otra ocasión el análisis temporal de la historia de los conceptos. Un camino para relacionar ambos proyectos teóricos de Koselleck podría comenzar por tener en cuenta que en Futuro pasado se da también una clasificación formal de "tres modalidades temporales de la experiencia”: irreversibilidad de los acontecimientos, repetibilidad de los acontecimientos y simultaneidad de lo anacrónico (Koselleck, 1993, p. 129), con el fin de mostrar los posibles elementos comunes y los elementos diferenciales entre las historias premodernas y la moderna historia absoluta (Koselleck, 1993, p. 128, 139).

3 La aplicación de estos modos formales a experiencias concretas modificables, así como la explicación de sus condiciones antropológicas dadas en la historia real se pueden observar en el apartado titulado "La historia de los vencedores, una historiografía de los vencidos", donde se muestra cómo la experiencia única de los vencidos ha hecho posibles las distintas conquistas metodológicas de la historia que descubren condiciones de largo plazo como motivos explicativos del acontecer histórico. 


\section{A. ESTRUCTURAS TEMPORALES DE LA EXPERIENCLA HISTÓRICA}

Vivimos los aCONTECimientos como únicos, irreversibles y sorprendentes. Este es el primer dato de la experiencia del tiempo, algo, dice Koselleck (2001), "de lo que cualquiera tiene experiencia en su propia biografía" (p. 36). Esta primera forma de experiencia es "originaria", es decir, necesaria a toda biografía y a toda historia (Koselleck, 2001, p. 50).

Pero el análisis de la unicidad como primer dato de la experiencia lleva a un segundo modo de experiencia, pues lo único solo emerge, como dato de vivencia, por contraste con lo regular. Desde lo más cotidiano, lo recurrente es presupuesto de lo único: cada mañana, a la misma hora, llega el cartero para traer noticias únicas; quien quiere decir algo nuevo, debe hacerlo desde un patrimonio lingüístico dado, y así podemos seguir con muchos otros ejemplos cotidianos (Koselleck, 2001, p. 37). Pero los fenómenos de recurrencia no son fijos, sino también variables. La teoría de los estratos del tiempo permite analizar esas distintas velocidades en el cambio, para dar cuenta del proceso de aquel cambio más profundo, sin limitarse al recuento de los procesos y cambios más visibles.

Los fenómenos de recurrencia no son, pues, estructuras fijas y ahistóricas, sino que se descubren por contraste u oposición con lo que aparece como único. La unicidad se encuentra en la vivencia de una sorpresa, es decir, cuando algo sucede distinto de como se esperaba. Lo que surge en esta brecha entre una expectativa y un acontecimiento es lo nuevo, "un mínimum temporal que se genera entre el antes y el después" (Koselleck, 2001, p. 39). "Lo nuevo" y "lo repetible” son analizados aquí por las relaciones que suponen las categorías temporales de "experiencia" y "expectativa": lo nuevo es la vivencia de un desfase entre estas, mientras que lo repetible, diríamos, como un paso continuo de la una a la otra. La expectativa se proyectaba, en efecto, desde un espacio de experiencia dado por algún patrón de recurrencia que le permite anticipar algo del futuro. Al romperse la continuidad entre el antes de la experiencia y el después proyectado, surge una nueva experiencia y se abre un nuevo horizonte ${ }^{4}$.

4 Así es como Koselleck determina el cambio de la experiencia moderna, desde el hilo conductor del cambio dado respecto de las perspectivas de futuro premodernas (Koselleck, 1993, pp. 27, 31). En esto encontraríamos otro empalme entre la teoría de los estratos del tiempo y la historia de los conceptos de Futuro pasado, en cuanto dichos estratos son analizados desde las categorías de "espacio 
El análisis puede ahora dar un paso adelante. Más allá de la sorpresa de lo novedoso y la ampliación de la experiencia (como acopio de sorpresas posibles) hay un tercer nivel temporal, determinado por un límite biológico de la experiencia humana: a mayor experiencia acumulada, menor capacidad de sorpresa, hasta que la resistencia frente a las sorpresas termina bloqueando la experiencia posible. Sobre esa finitud en el curso de una vida se delimitan los tiempos históricos. Este límite biológico va de lo individual a los ámbitos más amplios de la historia política o económica. Aquí cobra sentido hablar de unidades generacionales de individuos que viven en el mismo tiempo, entre disposiciones sociales y experiencias similares (Koselleck, 2001, p. 40). Así, moviéndose siempre en un plano de análisis antropológico de la experiencia, Koselleck da un paso más para mostrar un tercer estrato temporal, más profundo: dada la anterior limitación biológica de las experiencias históricas, se llega al estrato de los "tiempos históricos que sobrepasan la experiencia de individuos y generaciones". Por ejemplo, los comportamientos mágicos, las actitudes religiosas o científicas, o las concepciones de mundo que "se repiten en ritmos más lentos que el cambio que es directamente experimentable en el transcurso de unas generaciones" (Koselleck, 2001, p. 41).

El anterior análisis descubre tres "estructuras temporales de la experiencia histórica” (Koselleck, 2001, p. 56): la vivencia de lo nuevo, la acumulación de experiencias repetibles y la trascendencia de estas en fenómenos de largo plazo que escapan a la experiencia individual. La relación de dichas capas temporales con sus respectivos modos de narración y escritura de la historia nos permitirá delimitar tres metodologías históricas de distinto alcance. Si toda historia trata de experiencias, propias o ajenas, "cabe suponer que los modos de contar las historias o elaborarlas metodológicamente pueden referirse a los modos de hacer, recoger o modificar experiencias" (Koselleck, 2001, p. 50).

de experiencia" y "horizonte de expectativa", categorías históricas formales que permiten establecer las condiciones de las historias posibles y tematizar tiempos históricos (Koselleck, 1993, pp. 335-337). 


\section{B. CONDICIONES METODOLÓGICAS PARA REGISTRAR, CONTINUAR Y REES- CRIBIR LA HISTORIA DE LA "NORMALIZACIÓN FILOSÓFICA"}

¿Cómo se Relacionan los tres modos de experiencia con la posibilidad de su narración e investigación históricas, para el caso de la historia de la filosofía en Colombia? Los tres niveles de experiencia que hemos visto son, en palabras de Koselleck (2001), “aspectos comunes mínimos de toda metodología histórica que no puede renunciar al registro único, a la escritura acumulada y a la reescritura una y otra vez evocada" (p. 57).

Veremos entonces cómo se da este triple nivel en el uso del concepto de normalización filosófica en Colombia. Partimos de la premisa de entender este término como un concepto histórico en dos sentidos relacionados: el primero, en cuanto describe una experiencia de hacer filosofía en Colombia; el segundo, en cuanto ha servido para escribir y periodizar la historia de dicha experiencia. Un breve recorrido cronológico por los sentidos que ha ido tomando este concepto pondrá de relieve la pertinencia de recorrer esos cambios de sentido desde la teoría de los estratos del tiempo.

\section{Esbozo cronológico del término "normalidad" en el mundo hispánico y en Colombia}

LA IDEA DE NORMALIZAR LA FILOSOFía en el mundo hispánico proviene al parecer de José Ortega y Gasset, quien quiso superar el atraso científico y filosófico de España, haciendo frente al fenómeno de la decadencia española. En su artículo "España invertebrada", de 1921, remplaza el término decadencia por el de anormalidad (Cruz Vélez, 1991, pp. 80-82). Desde entonces se encuentra el uso del concepto de anormalidad para explicar la marginalidad y el anacronismo filosófico e histórico de España y, por extensión, del mundo hispánico (Cruz Vélez, 1991, p. 85). De ahí también que Ortega emprendiera la fundación de la Revista de Occidente, en 1923, para normalizar y poner al día todo el mundo hispano. La idea de alcanzar una "normalidad" cultural se entendió entonces como la tarea de occidentalizar Hispanoamérica (Cruz Vélez, 1991, p. 85).

Uno de los abanderados de la empresa de normalización orteguiana en América Latina fue Francisco Romero, desde Argentina. Fundador de la editorial 
Losada y profesor universitario, hizo más énfasis en los aspectos institucional y cultural de la "normalidad filosófica". Esta, según el maestro argentino, constituía una nueva etapa de la filosofía en Iberoamérica, consistente en el ejercicio de la filosofía como función ordinaria de la cultura, es decir, como expresión de madurez cultural y como actividad regular, habitual y común, ya no ejercida por personajes aislados a la vida normal de la cultura. La función cultural implicaba, pues, no solo una formación y vocación filosóficas, sino también contar con los medios institucionales necesarios a una comunidad de oficio (Fornet, 2000, pp. 120-121). En otras palabras, la normalidad filosófica se presentó como la consolidación de un oficio teórico, en que la filosofía, como cualquier otro oficio, requería del aporte conjunto de mentes no extraordinarias ni solitarias, que fueran conformando cierto "clima filosófico" (Romero, 1951, p. 150). Por esta característica se define, aún hoy, la normalización desde su aspecto institucional, como profesionalización de la filosofía en Latinoamérica.

Pero Romero daba testimonio de otro hecho que sugería un aspecto más de la normalidad: dada la creciente constitución de grupos filosóficos en los distintos países, Romero invitaba a reflexionar sobre temas como "el curso total de la cultura en estas tierras, sobre la peculiaridad del 'hecho' americano" (Romero, 1951, p. 155). La normalización no solo tenía que ver, entonces, con la apropiación de la tradición filosófica universal (occidentalización), o con la instalación de un oficio entre otros (institucionalización) sino que además, en el contexto latinoamericano, implicaba una toma de conciencia sobre el valor del filosofar en la conformación cultural de los pueblos (Tovar, 2009a, p. 454). Para Romero, ciertamente, la cultura es inconcebible sin filosofía o, en otros términos, la filosofía es la cultura misma en cuanto idea o problema (Romero, 1957a, p. 123). Se entiende así que, según él, el momento de la "normalidad filosófica" consistiera en un momento de madurez cultural en el que "la cultura de nuestros países se eleve a la conciencia filosófica” (Romero, 1957c, p. 9). Las principales culturas humanas tienen su raíz en una tendencia común al hombre y a la filosofía: la tendencia hacia el absoluto (Romero, 1957b, pp. 46-52).

El caso colombiano presenta ciertas particularidades en este proceso. En primer lugar, desde la historia de la filosofía en Colombia no se habla de una "etapa de normalidad filosófica" sino de una "normalización filosófica". Mientras en las otras historias latinoamericanas se habla de "normalidad" como del acceso a un 
momento de madurez alcanzado (Romero, 1951; Larroyo, 1989, p. 33, 115), en el caso colombiano es más común el uso del término "normalización" como referencia a un proceso inacabado (Pachón, 2011, pp. 129-140, 88; Giraldo, 2010; Botero, J. 2009; Tovar, 2009b; Marquínez, 2005; Hoyos, 1999), que arranca en los años cuarenta del siglo XX, pero abre un período de término indefinido que al parecer se extiende hasta nuestros días. Su alcance histórico sigue, pues, indeterminado.

Relacionado con lo anterior, aquel estado de madurez alcanzado en otros países durante las primeras tres décadas del siglo XX, en el caso colombiano se ha presentado con más modestia como un proceso iniciado relativamente más tarde, con la República Liberal, interrumpido por condiciones políticas adversas en los años cincuenta y solo continuado y parcialmente consolidado en las últimas tres décadas del siglo XX (Pachón, 2011; Botero, 2009; Marquínez, 2005; Salazar R., 1986).

También en Colombia, sin embargo, la normalización se presentó en su doble aspecto institucional y cultural. Como ha indicado el profesor Leonardo Tovar, la creciente fundación de las revistas universitarias y filosóficas, entre las décadas de los años 30 y 40, no solo apuntaba a difundir el saber académico, sino que obedecía también a una aspiración cultural, según la proclamó la Revista Colombiana de Filosofía en 1948, "Colombia necesita de la filosofía", como disciplina que le da contenido a toda cultura (Botero, 1948, pp. 3-6, 57; Tovar, 2009a p. 446). En las últimas décadas del siglo XX, sin embargo, estas intenciones culturales de la normalización filosófica parecen haberse silenciado, al menos a juzgar por el concepto de "normalización" que presentan las más recientes historias de la filosofía en Colombia. ¿Qué determinó este cambio? ¿Cuál fue el camino por el cual la "normalización filosófica", que al parecer movilizó las inquietudes y aspiraciones de toda una generación de filósofos colombianos, dejó de sostener la bandera de una misión cultural y se revistió solamente de un sentido institucional de la filosofía como profesión y occidentalización? Esta sería una pregunta clave para emprender la investigación histórica del concepto de "normalización filosófica" desde una teoría de la historia que pueda dar cuenta de los cambios de experiencia que han acompañado a dicho concepto.

Pues bien, el anterior recorrido cronológico muestra cómo el concepto en cuestión carga con una serie de notas o contenidos que se le han ido incorporando 
en el transcurso de ya casi un siglo: occidentalización, actualización, modernización, secularización, mayoría de edad, toma de conciencia cultural, autonomía disciplinar, profesionalización, institucionalización. Un análisis crítico del concepto de normalización filosófica ha de recorrer estas variaciones de sentido y preguntarse por los modos de experiencia que dan cuenta de ellas. A continuación se presentan, pues, los tres niveles en que podrían analizarse las distintas notas de este concepto. Habrá que prestar atención también a las mínimas aproximaciones metodológicas que corresponderían a cada nivel de experiencia. Con esta propuesta quiero sugerir una aproximación ordenada y crítica a las referencias dispersas que se encuentran en la bibliografía de la filosofía en Colombia del siglo XX.

\section{Aproximación a los estratos históricos de la "normalización filosófica" en Colombia}

DE ACUERDO CON LOS TRES NIVELES de experiencia expuestos, la investigación histórica tiene también tres niveles de profundidad: el registro de lo único, la continuación o acumulación de experiencias repetidas y la reescritura de la historia.

En el nivel del registro histórico, ha sido un lugar común presentar la normalización como un momento de ruptura, único en la historia de la filosofía en Colombia, en contraste con el pasado filosófico.

En cuanto a los requerimientos metodológicos mínimos de esta aproximación, el registro histórico de la normalización responde a dos preguntas: ¿cómo sucedió y por qué sucedió así y no de otra manera?5 Para responder a lo primero,

5 Toda historia pretende una novedad: el registro de lo único, imprevisto y sorprendente (Koselleck, 2001, p. 57). Para ello, sin embargo, no basta la narración de las experiencias en la vida cotidiana. Más allá de lo anecdótico, para conocer la unicidad de un acontecimiento se tiene que atender a dos preguntas: “¿qué ocurrió y cómo se llegó a ello?”. La respuesta a estas preguntas pide el seguimiento de unos caminos mínimos de investigación, descubiertos desde Heródoto y Tucídides, independientes del vehículo o la metodología concreta con que estos caminos sean recorridos. El primer camino es la colección de datos que describa cómo fue, o cómo ocurrió ese acontecimiento: ordenar los datos en una diversa profundidad temporal, según ellos provengan de testigos visuales, testigos orales o testimonios escritos (Koselleck, 2001, p. 59). Pero falta todavía conocer el carácter único del acontecimiento descrito: ¿por qué sucedió así y no de otra manera? La explicación de cómo fue posible una experiencia única involucra dos enfoques distintos, uno de medio plazo que describe causas y consecuencias coyunturales, otro de largo plazo que confronta el acontecimiento único con presupuestos duraderos (motivos como la patología del poder humano, la ceguera de los hombres, etc.) (Koselleck, 2001, p. 60). Ya acudiendo a los dioses, al destino, el deseo de poder, la 
se requiere colectar los datos que permitan describir el fenómeno. Los trabajos del profesor Sierra Mejía sobre las tendencias de la filosofía en el siglo XX (Sierra, 1982, p. 80), o los sondeos bibliográficos de la filosofía correspondientes a este período (Molano, 2006) pueden dar una idea de este tipo de trabajo. Pero, además de la descripción, falta todavía explicar su carácter único, por qué sucedió así y no de otra manera, ya por referencia con su pasado en una cadena causal, ya por su interpretación a través de las condiciones de medio o largo plazo que la hicieron posible. En cuanto enfoque causal, varios comentaristas hablan de la normalización como un momento de ruptura respecto del pasado escolástico (Botero, 2009, p. 1194, 1988; Sierra Mejía, 1985, pp. 9-11; 2009, p. 164; González, 2009; Ospina, 2007) o como consecuencia de una secularización del pensamiento dentro de la sociedad (Pachón, 2011, p. 59; Naranjo, 1976, p. 77; 1988); en cuanto al segundo enfoque, de medio y largo plazo, es usual presentar la normalización como una ganancia de autonomía disciplinaria, en contraste con la anterior subordinación política o religiosa de la filosofía (Botero, 2009; Tovar, 2009a, 2009b; Salazar, F. 1994: 93; Jaramillo Uribe, 1987; Sierra Mejía, 1985).

Mas esta "ruptura" o "novedad" respecto del pasado es solo una parte de verdad que no debe entenderse de manera unilateral, sino sobre un fondo de continuidad. El mismo Francisco Romero negaba que la normalidad fuera un punto cero que diera la espalda a los antecedentes filosóficos de Latinoamérica. Al contrario, el interés por la historia de la filosofía local era uno de los fenómenos que evidenciaban, para él, el arribo a la normalidad (Romero, 1951, p. 154). Romero sitúa la normalidad filosófica como la tercera de tres etapas que ha recorrido la filosofía en Latinoamérica, una nueva época que, sin embargo, es deudora de su época anterior: "si la filosofía es ahora tarea natural y aun habitual entre nosotros (...) es porque tenemos a nuestra espalda la generación insigne de los fundadores", los insignes solitarios (Romero, 1957c, p. 14). Hemos visto al comienzo de este trabajo que este hablar de unos "fundadores" de la normalidad filosófica im-

fortuna, el Dios cristiano, las condiciones de producción, los determinantes económicos, la acumulación de datos, etc., se trata siempre de una apelación a instancias últimas de repetibilidad para interpretar experiencias primarias de lo nuevo. Estas explicaciones se han ido modificando, pero "se mantiene la estructura formal de procesamiento metodológico de la experiencia": el análisis de una refracción temporal, del desfase entre un antes y un después, a través de la comparación entre lo único y lo duradero (Koselleck, 2001, p. 62). 
plica una serie de exclusiones históricas que no deben descuidarse (Fornet, 2000). En el segundo estrato veremos que un marco más amplio de continuidad se podrá tender, por ejemplo, desde un concepto analógico de filosofía, mucho menos restringido y excluyente.

En el caso colombiano, por ejemplo, hace falta examinar en qué ámbitos se habló de una ruptura o novedad durante la década de los años cuarenta y cincuenta. En efecto, la novedad se vio también desde el trasfondo de continuidad con una tradición de pensamiento; pero tampoco se promulgó una idea de normalidad filosófica excluyente de otras áreas de pensamiento. En 1946, Luis Eduardo Nieto Arteta hacía alusión a "la actual transformación de la cultura colombiana, la nueva poesía, la nueva filosofía, la nueva ciencia jurídica” (Nieto, 1978a, p. 228), y veía esta transformación como paralela a la de las otras naciones latinoamericanas (Nieto, 1978b, p. 237). Pero Nieto también buscaba situar este nuevo momento en una perspectiva acumulativa, al rescatar lo que consideraba las "adquisiciones irrevocables" del pensamiento jurídico colombiano (Nieto, 1978c).

Toda experiencia de novedad debe registrarse, pues, sobre el trasfondo de un segundo nivel: la historia como continuación y acumulación de experiencias. Así como la anécdota no era suficiente para el registro de historias únicas, en el segundo estrato no basta la mera acumulación cuantitativa de experiencias, pues siempre están latentes el riesgo del olvido y la sobrevaloración de las propias vivencias como únicas fuentes de experiencia. Para poder hablar de un incremento continuado de la experiencia se necesita del ordenamiento sistemático de lo diacrónico basado en una ampliación retrospectiva del tiempo (Koselleck, 2001, p. 63) $)^{6}$. Jaime Jaramillo Uribe, en un artículo de 1954 titulado "Tradición y problemas de la filosofía en Colombia”, partía de mostrar los problemas, temas e intereses que legaron los pensadores colombianos de finales del siglo XIX. Problemas que ocuparían el centro de atención del siglo XX como, por ejemplo, la desconfianza respecto del progreso, la potencia ilimitada de la ciencia, la paz que traería el industrialismo en la era positiva. Si bien las tres primeras décadas del siglo XX se

6 La historia, a este nivel de la experiencia temporal, se escribe y continúa con la recolección de todos los conocimientos que se han adquirido mediante un perfeccionamiento metodológico. Fue así como, con el curso acumulativo de los tiempos, se fueron desarrollando métodos para dar cuenta del creciente entrelazamiento espacial hacia una historia mundial y de la creciente concordancia temporal hacia una historia singular (Koselleck, 2001, p. 67). 
caracterizarían, según él, como afilosóficas y más concentradas en el progreso material del país, Jaramillo observa que a mediados de los años 30 "empezamos a sentir una transformación que puede ser de alcances verdaderamente fecundos", "un renacimiento y una superación de nuestra pequeña pero cierta tradición filosófica” (Jaramillo Uribe, 1954, p. 75). Se ve aquí que Jaramillo Uribe no hablaba de una ruptura con la tradición, sino que se situaba en el nivel de una continuación de la historia, al hablar de un renacimiento y superación de dicha tradición?

Pero aun en este segundo nivel de profundidad, el concepto de normalización presenta notas que han permitido trazar un hilo de continuidad no solo referido al pasado, sino también proyectado hasta nuestros días. En primer lugar, su historia continuada es posible por el transcurso efectivo de un mínimo de generaciones. Las historias acumuladas de estas generaciones pueden compararse por yuxtaposición o por sucesión. Tiene lugar aquí la comparación generacional de la normalización como proceso continuo, en dos enfoques, uno sincrónico y otro diacrónico ${ }^{8}$.

7 Llama la atención también que Jaramillo Uribe le dé tanta importancia al legado de F. Romero, pero no haga referencia alguna al término "normalidad". Toma evidentemente varios de sus aspectos, pero no lo utiliza para caracterizar el nuevo momento de la filosofía, prometedor y fecundo. $\mathrm{Si}$ se observa con detenimiento, en Colombia las referencias a la "normalidad" como momento nuevo $y$ de ruptura respecto del pasado no fueron registradas durante esa misma época, sino que al parecer surgieron durante el movimiento historiográfico de los años ochenta.

8 Para el caso de la integración acumulativa de la historia en una historia mundial y singular, Koselleck distingue estos dos enfoques. Primero, la adición de nuevas experiencias por transcripción, cuando la historia fue una rama de la retórica y servía para procesar y estabilizar experiencias que pueden reiterarse (Koselleck, 2001, p. 63); después, la historia se montó en un plano superior, cuando Polibio tematizó la unidad de historias diferentes a través de la geografía, un contexto general que escapaba de la vivencia individual (Koselleck, 2001, p. 64). Con esta ganancia se desarrolló también la necesidad de hacer una comparación sincrónica de diversos acontecimientos, mediante una datación unitaria que, finalmente, llevó a establecer una cronología común a todas las culturas heterogéneas. Todos estos procedimientos comparativos tienen como su condición de posibilidad el segundo modo de experiencia, en cuanto "presuponen un mínimo de historias ya transcurridas y diferenciables por yuxtaposición o sucesión”. Los métodos comparativos y las tipologías que se apoyan en estos mismos presupuestos determinan la repetibilidad de experiencias y de evoluciones previsibles (Koselleck, 2001, p. 65). Pero, además de eso, la continuación de la historia es experiencia acumulada según reglas de sucesión puramente diacrónicas, es decir, puede tener una interpretación de largo alcance. Un ejemplo de esas reglas diacrónicas es el principio aristotélico, introducido a la historia por Polibio y Tácito, de que causas pequeñas pueden tener grandes efectos. Otra regla de procesamiento diacrónico consiste en el uso de métodos analógicos que permiten interpretar un caso particular desde contextos a largo plazo: por ejemplo, cuando desde la idea de una providencia divina, o la interpretación progresiva de avances o cambios sociales, se usa el sentido plural de una fuente escrita para situar el caso particular (Koselleck, 2001, p. 66). 
En primer lugar, el enfoque sincrónico daría una muestra de experiencias mutuamente comparables, por ejemplo, las referidas a la normalización como proceso de profesionalización de la filosofía. Así lo ha hecho Juan José Botero al dividir el proceso de la normalización en tres generaciones, comparando currículos de enseñanza y resultados institucionales de cada una. Desde esta perspectiva, sería posible y atractiva la comparación de experiencias yuxtapuestas dentro de una misma generación; esto es, poner en discusión distintas experiencias de profesionalización testimoniadas en Rafael Carrillo, Cayetano Betancur y Danilo Cruz Vélez, o evidenciar el contrapunto de sus ideas sobre la relación entre filosofía y universidad. En efecto, si bien los miembros de esta generación participaron de las tareas profesoral y profesional definidas por Romero, también es un hecho que cuestionaron dichas labores, distinguiendo entre filosofía y filosofar, y resaltando el valor de trabajar sobre problemas filosóficos por encima de la pretensión de construir sistemas, bajo la sospecha de los riesgos que entrañaba la filosofía profesional. Carrillo oponía la filosofía como profesión lucrativa a la filosofía como vocación desinteresa por el saber (Carrillo, 1986, p. 13). Cruz Vélez insistía en recordar el riesgo del filósofo burócrata, que sacrifica su libertad por estar al servicio de una institución (Cruz Vélez, 1991, p. 105, 169-177, 201; 1977, pp. 99, 219). Betancur, por su parte, dudaba sobre la filosofía profesional por la marginalidad social en que esta puede caer (Betancur, 1988a, p. 231; Gómez, 2008, p. 218)99.

En segundo lugar, el enfoque diacrónico permitiría hacer una interpretación progresiva de momentos distintos de la filosofía en Colombia, conforme a algún concepto de largo alcance de filosofía. Guillermo Hoyos, por ejemplo, hacia finales de los años 90 veía el ingreso de la filosofía moderna y cambio de actitud hacia la filosofía práctica (así entiende la normalización), como un proceso en el cual tres generaciones de filósofos han contribuido. Para él, la normalización iniciada en los años 40 extiende sus alcances más allá de sus iniciadores, sin término fijo, en un viraje hacia la filosofía práctica como "preocupación recurrente, nece-

9 La universidad debe responder principalmente, según él, al problema de la formación de profesionales demasiado especializados, pero sin horizonte ético, pues la inteligencia sin límites morales corre el riesgo inminente de ser objeto de abuso, de ser utilizada para cualquier fin (Betancur, 1988b). Esta puesta en diálogo entre las vivencias de una misma generación sobre el aspecto profesional de la filosofía saca a la luz la variedad de posturas sobre una discusión que, como veremos, todavía en las últimas décadas se ha dado respecto del ejercicio de la filosofía en Colombia, tal como se recoge en el No. 104 de la revista Ideas y Valores y se ve en Restrepo, 2011, p. 14. 
sariamente nunca satisfecha, y hoy más que nunca pertinente" (Hoyos, 1999). Una preocupación de fondo que sigue en la línea de los escritores colombianos del siglo XIX, según lo señalaba Jaramillo Uribe. Al recordar la entrada del marxismo en las décadas del 60 y del 70, Hoyos rescata que esta orientación marxista era fundamentalmente ética (Hoyos, 1999). La tendencia marxista continuaba asi en la línea del cambio de actitud hacia la filosofía práctica iniciado, según Hoyos, en los años 40. Otra propuesta de interpretar todo el proceso histórico de la filosofía en Colombia se halla en Leonardo Tovar (2009b), en línea con la hermenéutica analógica (Marquínez y Beuchot, 2005), cuando habla de un concepto analógico de filosofía: la filosofía como instancia crítica dentro de una sociedad. Desde tales conceptos de largo alcance, las lecturas de la normalización como proceso acumulativo pueden abrir el camino hacia la investigación de la relación entre filosofía y cultura, tal como se pretendía en la década del 40.

Finalmente, una reescritura de la historia ${ }^{10}$ de la "normalización filosófica" será posible mostrando la multiplicidad de significados que ha tomado el concepto de "normalización" en las historias escritas, así como el desfase entre los significados que han ido predominando en ellas y las pretensiones iniciales que venían expresadas bajo la idea de filosofía o la de normalización. En los años 80 la historia de la filosofía en Colombia tomó un nuevo enfoque desde una preocupación por vincular la filosofía con la praxis. La demanda de autenticidad y crítica social respecto de la filosofía se pronunció como un reproche a la "generación

10 Kosselleck explica que la experiencia de Tucídides puso de presente las condiciones de toda reescritura de la historia. Al confrontar todos los discursos y las crónicas de la guerra del Peloponeso, Tucídides sacó a la luz una experiencia antropológica originaria como condición de todo cambio de experiencia (dado en los tres niveles): señaló la contradicción entre la realidad y el lenguaje, entre la historia efectiva y su interpretación textual. Desde entonces, esa distinción todavía inagotable es constitutiva de toda experiencia histórica y hace posible cualquier revisión, reinterpretación y reescritura de lo que se dice sobre los hechos; así, debido a la multivalencia lingüística, la verdad de la historia no viene garantizada por el recurso a testimonios orales inmediatos (que era el mínimo de continuidad de las experiencias pasadas) (Koselleck, 2001, pp. 72-74). Teniendo como eje la diferencia entre discurso y acción como condición metahistórica de toda reinterpretación, se dan tres usos del testimonio escrito como posibilidades de reescritura (Koselleck, 2001, p. 76): (a) el descubrimiento de nuevos testimonios obliga a elegir entre las fuentes; (b) el surgimiento de nuevas cuestiones que llevan a nuevos testimonios; por ejemplo, al ir más allá de las fuentes orales, para fijarse en documentos, actas, inscripciones, etc. La tradición aparece entonces bajo una nueva luz; (c) la nueva lectura e interpretación de los testimonios que ya se tienen. Esta nueva lectura puede darse, o bien para redescubrir un supuesto sentido originario (ej., el caso del protestantismo con su vuelta a las Escrituras), o bien para deducir afirmaciones no pretendidas por los autores. 
normalizadora" de los años 40, adjudicándole un enfoque repetitivo y devoto por el pensamiento europeo (Tovar 2009b; Molano, 2006; Hoyos, 1999). Así, las divergencias entre la historia de los años 80 y la producción filosófica de las décadas del 40 al 60 abren una serie de preguntas que podrían trazar el camino a una reescritura de la historia de la filosofía en Colombia. El contraste entre los conceptos de filosofía y normalización en los dos tipos de fuentes podría dar testimonio de un cambio de experiencia dado en el campo de la filosofía, entre el segundo y el tercer tercio del siglo XX. La historia de los años 80 reconoció el aporte institucional y profesional de los primeros "normalizadores", ¿pero su calificación de dicha actividad como "repetición devota" implica un olvido de las pretensiones culturales y de autenticidad que recaían sobre la filosofía en la década de los años 40? En el caso colombiano llama la atención que, en contraste con las décadas anteriores, sea en la década del 80 cuando el concepto de "normalización" es más usado y adquiere un sentido netamente institucional. ¿Se buscaba con ello señalar un proceso comenzado en los años cuarenta y sólo llevado a plenitud en los años 80 ?

El enfoque comparativo que venimos proponiendo puede dirigirse a momentos más recientes en la producción y autocomprensión filosófica colombiana. Es el caso de la discusión en torno al problema del "pensador profesional", recogida en el número 104 de la revista Ideas y Valores (1997), o de la polémica generada a raíz de la cuestión de la "torre de marfil" de los filósofos profesionales (Restrepo, 2011). ¿Qué se ha mantenido y qué ha cambiado de las notas de la normalización que hemos visto reunidas y que hemos tratado de discriminar en los anteriores niveles de análisis?, ¿cómo se han puesto en juego en las discusiones recientes?

Con lo anterior se pondría a prueba, en suma, si el concepto de "normalización" es pertinente para dar cuenta, o bien de un período ya clausurado de la filosofía en Colombia, o bien de un proceso todavía inacabado. Se espera abrir así el camino para señalar los cambios semánticos que ha tenido el concepto de normalización filosófica y los cambios de experiencia que puedan corresponder con dichas transformaciones. Pero todo cambio de experiencia implica también una posible pérdida de otras experiencias (Koselleck, 2001, p. 77). A través de una historia del concepto de "normalización filosófica", que precise cómo se mueve ese concepto, qué notas va tomando y qué conflictos va entrañando en virtud de su polivalencia y de los necesarios desfases entre sus distintos momentos y contextos 
de enunciación, tal vez pueda ponerse a prueba qué tanto potencial explicativo tenga el concepto en cuestión, si se trata de un concepto ya agotado y simplemente repetido como lugar común, o todavía puede dar buena cuenta del proceso de la filosofía en Colombia.

\section{Referencias}

Betancur, C. ([1954] 1988a). Expresión y formas de la filosofía en Colombia. Sociología de la autenticidad y la simulación (seguida de otros ensayos), 44. pp. 224-231

Betancur, C. ([1954] 1988b). La universidad y la responsabilidad intelectual. Sociología de la autenticidad y la simulación (seguida de otros ensayos), 44. pp. 208-221

Betancur, C. ([1948] 1969). Jaime Balmes. Filósofos y filosofías (pp. 99-108). Bogotá: Ed. Kelly.

Botero, A. (1948). Necesidad y posibilidad de la filosofía en Colombia. Revista colombiana de filosofia, 1 .

Botero, J.J. (2009). Filosofía en Colombia en el siglo XX: tres generaciones en busca de la normalización. Garrido et al. El legado filosófico español e hispanoamericano del siglo XX (pp. 1193-1205). Madrid: Cátedra.

Carrillo, R. (1986). Escritos filosóficos. Bogotá: USTA.

Cruz Vélez, D. (1977). Aproximaciones a la filosofía. Bogotá: Instituto Colombiano de Cultura.

Cruz Vélez, D. (1991). Tabula rasa. Bogotá: Planeta.

Fornet-Betancourt, R. (2000). Para un balance crítico de la filosofía iberoamericana en la llamada etapa de los fundadores. Anuario de Filosofía Argentina y Americana, 17, pp. 117-132.

Giraldo, J. (2010). Notas introductorias. J. Giraldo. (Ed.), Sobre politica. Artículos y fragmentos escogidos. Medellín: EAFIT.

Gómez García, J.G. (2008). Cayetano Betancur. N.A. Gil \& H. Ortiz, Pioneros de la filosofia moderna en Colombia (siglo XX). Bogotá: Ed. Ibáñez. 
González, I. (2009). Tradición y modernidad en el pensamiento de Cayetano Betancur. R. Sierra et al., República liberal: sociedad y cultura (pp. 483-518). Bogotá: Universidad Nacional.

Gutiérrez, R. (1989). Hispanoamérica: imágenes y perspectivas. Bogotá: Temis. Hoyos, G. (1999). Medio siglo de filosofía moderna en Colombia. Reflexiones de un participante. Revista de Estudios Sociales, 3.

Jaramillo Uribe, J. (1954). Tradición y problemas de la filosofía en Colombia. Ideas y Valores, 3(9-10), pp. 58-82.

Jaramillo Uribe, J. (1987). La filosofía en el siglo XIX y principios del XX. Análisis, 45. Koselleck, R. (1993). Futuro pasado. Para una semántica de los tiempos históricos. Barcelona: Paidós.

Koselleck, R. (2001). Los estratos del tiempo. Estudios sobre la historia. Barcelona: Paidós. Larroyo, F. ([1969] 1989). La filosofia Iberoamericana. Historia. Formas. Temas. Polémica. Realizaciones. México, D.F.: Porrúa.

Marquínez. G. (2005). Historia del problema de la filosofía latinoamericana. G. Marquínez \& M. Beuchot, Hermenéutica analógica y filosofía latinoamericana (pp. 15-30). Bogotá: El Búho.

Molano et al., (2006). La historia de la filosofía en Colombia y el proyecto Sistema de información sobre la filosofía en Colombia (SIFCO). Hallazgos, 3(5), pp. 187-206

Naranjo, A. (1976). Generaciones colombianas. Bogotá: Breviarios Colombianos-Banco de la República.

Naranjo, A. (1988). Presentación. Sociología de la Autenticidad y la simulación (seguido de otros ensayos). Medellín: Ediciones Autores Antioqueños.

Nieto Arteta, L.E. ([1946] 1978a). Crítica a un programa de historia colombiana. Ensayos históricos y sociológicos (pp. 218-228). Bogotá: Instituto Colombiano de Cultura.

Nieto Arteta, L.E. ([1950] 1978b). Desarrollo histórico de la cultura colombiana. Ensayos históricos y sociológicos (pp. 229-238). Bogotá: Instituto Colombiano de Cultura.

Nieto Arteta, L.E. ([1938] 1978c). De la legislación de Indias al nuevo código civil. Ensayos históricos y sociológicos (pp. 192-206). Bogotá: Instituto Colombiano de Cultura. 
Ospina, C.A. (2007). La soledad del pensador. Aleph, 143.

Pachón, D. (2011). Estudios sobre el pensamiento colombiano. Bogotá: Desde Abajo.

Palti, E. (2001). Introducción. Los estratos del tiempo. Estudios sobre la historia. Barcelona: Paidós.

Restrepo, R. (2011). ¿Dónde están los filósofos? Arcadia, 66.

Romero, F. ([1940] 1951). Sobre la filosofía en Iberoamérica. Filosofía de la persona y otros ensayos de filosofia (pp. 147-157). Buenos Aires: Losada.

Romero, F. ([1943] 1957a). La filosofía, la cultura y el hombre. Filósofos y problemas (pp. 119-128). Buenos Aires: Losada.

Romero, F. ([1945] 1957b). Hipótesis sobre las culturas. Filósofos y problemas (pp. 43-52). Buenos Aires: Losada.

Romero, F. ([1941] 1957c). Enrique José Varona. Filósofos y problemas (pp. 9-39). Buenos Aires: Losada.

Salazar, F. (1994). Filosofía y realidad. Estudios de filosofia, 10, pp. 79-99.

Salazar, R. (1986). Daniel Herrera o la fenomenología como filosofía de la ambigüedad. Escritos sobre fenomenología (pp. 11-34). Bogotá: USTA-Biblioteca Colombiana de Filosofía.

Sierra Mejía, R. ([1978] 1982). Temas y corrientes de la filosofía colombiana en el siglo XX. Cuadernos de filosofía latinoamericana, 12

Sierra Mejía, R. (1985). Prólogo. La filosofía en Colombia (siglo XX). Bogotá: Procultura

Sierra Mejía, R. (2009). Danilo Cruz Vélez (1908-2008) In memoriam. Ideas y valores, 58(139), pp. 161-177.

Tovar, L. (2000). La normalización filosófica en Colombia. Ensayo de crónica de las ideas. La filosofia del siglo XX: balance y perspectivas. Lima: Pontificia Universidad Católica del Perú.

Tovar, L. (2009a). Política y derecho en las ideas filosóficas durante la República Liberal. República Liberal: sociedad y cultura. Bogotá: Universidad Nacional de Colombia.

Tovar, L. (2009b). Las fundaciones de la filosofía latinoamericana. Sobre la filosofia y filosofia latinoamericana. Bogotá: USTA. 